\title{
Surtos de varicela em creches na Capela do Socorro em 2005
}

\author{
Varicella outbreak in the Capela \\ do Socorro, 2005
}

\begin{abstract}
Julio Zonzini Máximo de Carvalho', Thiago Ribeiro Rodrigues', Tatiana Tarules Azzi', Patrícia Carla Piragibe Ramos Burihan²
\end{abstract}

Carvalho JZM, Rodrigues TR, Azzi TT, Burihan PCPR. Surtos de varicela em creches na Capela do Socorro em 2005. Rev Med (São Paulo). 2007 jul.-set.;86(3):148-54.

RESUMO: A varicela é uma doença causada pelo vírus varicela-zoster. A varicela é extremamente transmissível, sendo transmitida de pessoa a pessoa, com risco maior em ambientes fechados, como creches, salas de aula e enfermarias. A vacina é segura e imunogênica em crianças, adultos saudáveis e em pacientes imunocomprometidos e mostra boa tolerabilidade com poucas reações colaterais possuindo eficácia de $80 \%$. O objetivo do trabalho foi analisar os surtos de varicela ocorridos em creches na região da Capela do Socorro, em 2005. O estudo realizado foi transversal, através do levantamento de dados dos surtos ocorridos em creches ao longo do ano de 2005, na região da Supervisão de Vigilância em Saúde (SUVIS) Capela do Socorro. Foram analisadas 36 instituições, cada uma com 1 surto de varicela, observando-se 617 casos em um total de 6845 alunos, com uma taxa de ataque $9,01 \%$. Os dados obtidos mostraram predomínio da faixa etária de 1 a 4 anos $\left(x^{2}=195.50\right)$, dos meses de inverno $(61.11 \%$ dos surtos) e maior número de surtos nas instituições próximas as UBS Veleiros, República e Mirna, respectivamente. A CEI José Molina Junior foi a que apresentou maior taxa de ataque $(38,46 \%)$. Devido ao alto grau de transmissibilidade da varicela e da extrema rapidez com que a mesma pode se disseminar em ambientes confinados como creches, é que se faz necessário um estudo referente a surtos em creches como as da Capela do Socorro.

DESCRITORES: Surtos de doenças. Varicela/transmissão. Creches. Estudos transversais.

Prêmio Oswaldo Cruz (POC).

1. Acadêmicos do $5^{\circ}$ ano do Curso de Medicina da Faculdade de Medicina da Universidade de Santo Amaro.

2. Médica, Professora da Disciplina de Saúde Coletiva da Faculdade de Medicina de Santo Amaro e Médica da Supervisão de Vigilância em Saúde da Capela do Socorro.

Endereço para correspondência: Julio Zonzini Máximo de Carvalho. Rua Jaricunas, 150. São Paulo, SP. CEP: 05053070. e-mail: juliozonzini@yahoo.com.br 


\section{INTRODUÇÃO}

varicela é uma doença causada pelo
vírus varicela-zoster (VVZ). Foi
confundida por muitos anos com a varíola até que em 1767 foi elucidada esta dúvida por Willian Herberden. A infecção aguda por este vírus causa a varicela, a qual é normalmente branda em crianças, principalmente em menores de 15 anos, porém extremamente grave em adultos e em pacientes imunocomprometidos. Pode ocorrer durante o ano todo, porém observa-se um aumento do número de casos no período que se estende do fim do inverno até a primavera (agosto a novembro), sendo comum neste período, a ocorrência de surtos em creches, pré-escolas e escolas. A varicela é extremamente transmissível. Cerca de $90 \%$ dos contactantes domiciliares expostos a pessoas com varicela podem adquirir a doença. $O$ risco é ainda maior em ambientes fechados como creches, salas de aula, enfermarias e consultórios. A transmissão se dá pessoa a pessoa e ocorre primariamente por contato direto com pacientes, por disseminação aérea de partículas virais (aerossóis) e, também, pode ocorrer por meio de contato com as lesões de pele.

O período de maior transmissibilidade iniciase dois dias antes do aparecimento das vesículas e vai até a fase de crosta. O período de incubação varia de duas a três semanas, com média de 14 a 16 dias. Após o período de incubação, a criança desenvolve simultaneamente febre e exantema, ocasionalmente ocorre o aparecimento isolado de uma ou mais vesículas, que pode preceder, em um a dois dias, o exantema generalizado. Normalmente o exantema inicia-se na face ou no couro cabeludo e dissemina-se rapidamente para o tronco, com menor acometimento de extremidades. As lesões iniciamse como máculas eritematosas que evoluem, em 8 a 48 horas, para todos os estágios, progredindo para vesículas e crostas. A desidratação das lesões leva à típica umbilicação central. Há tendência para as novas lesões surgirem em surtos, o total de lesões varia de duas ou três, nos casos muito leves.

O VVZ infecta membranas mucosas, pele, neurônios e estabelece uma infecção latente nos gânglios sensitivos, podendo a infecção retornar muitos anos depois causando a Herpes Zoster (HSV). Ao contrário do HSV, o vírus varicela zoster geralmente retorna somente uma vez, ocorrendo esta recidiva mais freqüentemente em pacientes imunocomprometidos e idosos. A doença pode ser prevenida através da utilização da vacina contra a varicela. Os países que adotaram a vacinação sis- temática das crianças contra a varicela observaram uma queda significativa do número de casos e de óbitos. Em 1975, a vacina contra a varicela foi desenvolvida no Japão, a partir do VVZ atenuado, tornando possível a prevenção da infecção em crianças saudáveis. A Vacina Oka/Merck foi licenciada para uso nos Estados Unidos em 1995. Antes de a vacina estar disponível, ocorriam por ano aproximadamente 11 mil hospitalizações e cerca de 100 óbitos devido à varicela neste país.

No Brasil, a vacina contra a varicela encontrase disponível desde 1998, nos Centros de Referência para Imunobiológicos Especiais (CRIES), em três apresentações comerciais, todas elas utilizando a cepa japonesa original de Oka. Esta está indicada para todas as crianças acima de 1 ano de idade além de adolescentes e adultos susceptíveis, que não tiverem contra-indicação. A vacina está contraindicada em indivíduos que tenham apresentado reação alérgica grave a uma dose prévia ou a qualquer um de seus componentes. Além disto, assim como todas as vacinas de vírus atenuado, também está contra-indicada durante a gravidez e em pessoas com imunodeficiência. Não está indicada em menores de 1 ano de idade em função da baixa eficácia nesta faixa etária (interferência dos anticorpos maternos transferidos pela placenta) e pela falta de informação quanto à segurança de uso neste grupo.

A vacina é segura e imunogênica em crianças, adultos saudáveis e em pacientes imunocomprometidos, mostra boa tolerabilidade, com poucas reações colaterais locais; possui eficácia em torno de $80 \%$ na proteção contra qualquer forma da doença e de $98 \%$ na prevenção de doença moderada ou severa.

No Brasil, a varicela não é uma doença de notificação compulsória e os dados existentes são esparsos e pouco representativos, porém vale lembrar a importância de se notificar surtos (ocorrência de dois ou mais casos de varicela, confirmados clinicamente, restritos a um mesmo estabelecimento, num período menor do que quatro semanas) ao SINAN (Sistema Nacional de Agravos de Notificação - Boletim de notificação de surtos).

\section{OBJETIVOS}

\section{Objetivo geral}

Analisar os surtos de varicela ocorridos em creches na região da Capela do Socorro, em 2005. 


\section{Objetivos específicos}

Comparar os surtos de varicela de acordo com a faixa etária mais atingida, o mês de ocorrência e a Unidade Básica de Saúde mais próxima a cada instituição.

Calcular as taxas de ataque de cada creche.

\section{CASUÍSTICA E MÉTODO}

Foi realizado um estudo transversal, através do levantamento de dados dos surtos (figura 1) ocorridos em creches ao longo do ano de 2005, na região da Supervisão de Vigilância em Saúde (SUVIS) Capela do Socorro, que abrange os distritos administrativos de Socorro, Cidade Dutra e Grajaú.

Foram analisadas todas as instituições de ensino para crianças de 0 a 9 anos de idade, que notificaram surtos de varicela para a SUVIS Capela do Socorro, sendo encontrados 36 surtos, totalizando 617 casos. As instituições analisadas foram representadas por estabelecimentos de administração direta da Prefeitura, como Centro de Educação Infantil (CEI), Escola Municipal de Educação Infantil (EMEI) e Centro Educacional Unificado (CEU); e por creches conveniadas, como Unidades Recreativas, Escolas, Centros Sociais Comunitários e Fundações.

Todos os surtos foram obrigatoriamente notificados para a Unidade Básica de Saúde mais próxima ao estabelecimento, compreendendo as UBS Jardim Eliane, Jardim Mirna, Jardim Varginha, Parque Residencial Cocaia, Jardim República, Jardim Castro Alves, Veleiros, Jardim Icaraí Quintana, Jordanópolis,
Ambulatório de Especialidades Dr. Milton Aldred e Ambulatório de Especialidades Jardim Cliper.

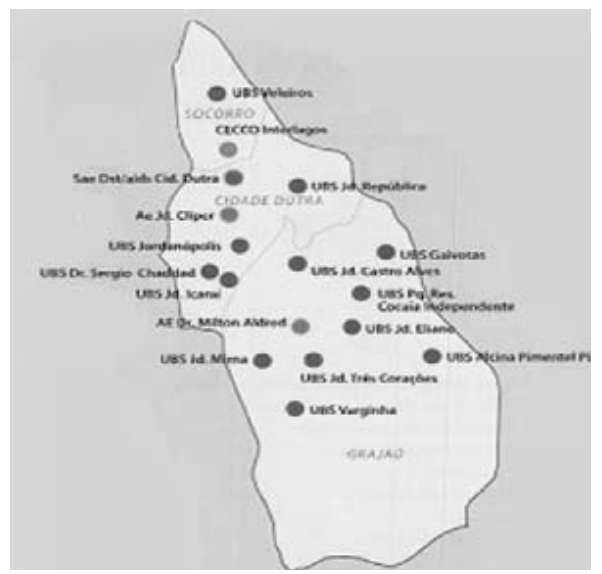

Figura 1

As variáveis analisadas no estudo foram: número de casos de varicela; número total de alunos da creche; vacinação de varicela prévia; quadro de varicela no passado; ocorrência de óbitos;

situação de suscetibilidade à doença; UBS mais próxima da instituição; data de início e término do surto dos casos de varicela, sendo o mês do surto definido pela data de início do primeiro caso.

Foi calculada a taxa de ataque (coeficiente de incidência), definida pela razão de casos novos pela população e multiplicado por cem, apresentando-se os resultados em porcentagem.

O método estatístico utilizado para estudar a relação entre as variáveis foi o teste do Quiquadrado, considerando- se significantes as que apresentaram $\mathrm{p}<0,05$.

\section{RESULTADOS}

Tabela 1. Instituições com surtos de varicela, por total de casos, taxa de ataque e UBS mais próxima, Capela do Socorro, 2005

\begin{tabular}{|c|c|c|c|c|}
\hline $\begin{array}{l}\text { Instituições } \\
\text { ADMINISTRAÇÃO DIRETA- CEI }\end{array}$ & $\begin{array}{l}\text { Total de alunos } \\
\text { das instituições }\end{array}$ & $\begin{array}{l}\text { Total de casos } \\
\text { de varicela }\end{array}$ & Taxa de ataque & UBS mais próxima \\
\hline CEI/COHAB Brigadeiro Faria Lima & 102 & 6 & $5,88 \%$ & AE Dr. Milton Aldred \\
\hline CEI Cel. Geraldo de Arruda Penteado & 116 & 07 & $6,03 \%$ & Jardim República \\
\hline CEI/CEU Cidade Dutra & 298 & 07 & $2,34 \%$ & AE Jardim Cliper \\
\hline CEI Doce União & 65 & 06 & $9,2 \%$ & AE Dr. Milton Aldred \\
\hline CEI Jardim Reimberg & 139 & 16 & $11,51 \%$ & AE Dr. Milton Aldred \\
\hline CEI Jardim Três corações & 169 & 03 & $1,77 \%$ & Jardim Mirna \\
\hline CEI Jardim Somara & 153 & 13 & $8,50 \%$ & Jardim Castro Alves \\
\hline CEI José Molina Junior & 130 & 50 & $38,46 \%$ & Jardim República \\
\hline CEI Mitiko Matsushita Nevoeiro & 226 & 30 & $13,27 \%$ & Jardim Mirna \\
\hline CEU/ CEI Navegantes & 298 & 34 & $11,4 \%$ & Pq. Residencial Cocaia \\
\hline CEI Nossa Senhora de Fátima & 102 & 28 & $27,45 \%$ & Veleiros \\
\hline CEI Núcleo III & 103 & 37 & $35,92 \%$ & Jardim Eliane \\
\hline CEI Parque América & 127 & 20 & $15,74 \%$ & Jardim Castro Alves \\
\hline CEI Parque Grajaú & 194 & 13 & $6,70 \%$ & Jardim Castro Alves \\
\hline CEI São Camilo & 159 & 45 & $28,30 \%$ & Pq. Residencial Cocaia \\
\hline CEU Três Lagos & 298 & 18 & $6,04 \%$ & Jardim Mirna \\
\hline CEI Veleiros & 145 & 03 & $2 \%$ & Veleiros \\
\hline
\end{tabular}


Continuação

\begin{tabular}{|c|c|c|c|c|}
\hline $\begin{array}{l}\text { Instituições } \\
\text { ADMINISTRAÇÃO DIRETA-EMEI }\end{array}$ & $\begin{array}{l}\text { Total de alunos } \\
\text { das instituições }\end{array}$ & $\begin{array}{l}\text { Total de casos } \\
\text { de varicela }\end{array}$ & Taxa de ataque & UBS mais próxima \\
\hline EMEl Ângelo Kretã & 525 & 05 & $0,95 \%$ & Jardim República \\
\hline EMEI Clara Nunes & 356 & 34 & $9,55 \%$ & Veleiros \\
\hline EMEI Sérgio Cardoso & 525 & 30 & $5,71 \%$ & Jardim República \\
\hline \multicolumn{5}{|l|}{ CONVENIADAS } \\
\hline Centro Social Comunitário Jardim Primavera & 224 & 41 & $18,30 \%$ & Jardim República \\
\hline Creche AMA I núcleo II & 126 & 28 & $22,22 \%$ & Jardim Eliane \\
\hline Creche Auri Verde & 72 & 09 & $12,50 \%$ & Varginha \\
\hline Creche Caetano Etiene & 142 & 04 & $2,81 \%$ & Jardim Mirna \\
\hline Creche Casinha São José & 213 & 15 & $7,04 \%$ & AE Jardim Cliper \\
\hline Creche Grão da Vida & 162 & 04 & $2,46 \%$ & Veleiros \\
\hline Creche Guarapiranga & 123 & 08 & $6,50 \%$ & Veleiros \\
\hline $\begin{array}{l}\text { Creche Mão Cooperadora } \\
\text { Creche Pequeno Príncipe } \\
\text { Creche Propame Assistencial }\end{array}$ & $\begin{array}{l}117 \\
245 \\
123\end{array}$ & $\begin{array}{l}12 \\
13 \\
08\end{array}$ & $\begin{array}{l}10,25 \% \\
5,30 \\
6,50 \%\end{array}$ & $\begin{array}{l}\text { Pq. Residencial Cocaia } \\
\text { Jardim Icaraí Quintana } \\
\text { Veleiros }\end{array}$ \\
\hline Escola Piemonte & 67 & 23 & $34,32 \%$ & Veleiros \\
\hline Escola Recreativa Anjinho Feliz & 99 & 05 & $5,05 \%$ & Jardim Mirna \\
\hline Fundação Marco Eduardo Brandão & 100 & 11 & $11 \%$ & Jordanópolis \\
\hline $\begin{array}{l}\text { Sociedade Beneficente e Equilíbrio de Interlagos } \\
\text { Unidade I }\end{array}$ & 471 & 17 & $3,60 \%$ & Jardim República \\
\hline $\begin{array}{l}\text { Sociedade Beneficente Equilíbrio de Interlagos } \\
\text { Unidade II }\end{array}$ & 266 & 07 & $2,63 \%$ & AE Jardim Cliper \\
\hline Unidade Recreativa Infantil Tia Suze LTDA & 65 & 07 & $10,76 \%$ & Varginha \\
\hline Total & 6845 & 617 & $9,01 \%$ & \\
\hline
\end{tabular}

Fonte: SUVIS - Capela do Socorro.

Tabela 2. Surtos de varicela segundo faixa etária, total de casos, total de alunos, ocorrência de óbito, taxa de ataque e suscetibilidade, Capela do Socorro, 2005

\begin{tabular}{lcccccrr}
\hline Faixa etária & $\begin{array}{l}\text { Total de alunos } \\
\text { das creches }\end{array}$ & $\begin{array}{l}\text { Total de casos } \\
\text { varicela }\end{array}$ & Varicela anterior & $\begin{array}{l}\text { Vacina de varicela } \\
\text { prévia }\end{array}$ & Óbito & Taxa de ataque & Suscetível \\
\hline $0-1$ & 212 & 13 & 26 & 1 & 0 & $6,13 \%$ & 172 \\
$1-4$ & 4073 & 524 & 1302 & 147 & 0 & $12,86 \%$ & 2100 \\
5 & 1429 & 69 & 584 & 94 & 0 & $4,82 \%$ & 682 \\
$6-9$ & 1131 & 11 & 471 & 10 & 0 & $0,97 \%$ & 639 \\
\hline Total & 6845 & 617 & 2383 & 252 & 0 & $9,01 \%$ & 3593 \\
\hline
\end{tabular}

Fonte: SUVIS Capela do Socorro.

Tabela 2a. Surtos de varicela por total de casos por faixa etária, Capela do Socorro, 2005

\begin{tabular}{lllll}
\hline Faixa etária & Casos & Não casos & $\begin{array}{c}\text { Total de } \\
\text { alunos }\end{array}$ & Taxa de ataque \\
\hline $0-1$ & 13 & 199 & 212 & $6,13 \%$ \\
$1-4$ & 524 & 3549 & 4073 & $12,87 \%$ \\
5 & 69 & 1360 & 1429 & $4,83 \%$ \\
$6-9$ & 11 & 1120 & 1131 & $0,97 \%$ \\
\hline Total & 617 & 6228 & 6845 & $9,01 \%$ \\
\hline
\end{tabular}

$X^{2}$ calc $=195.50 \quad(p<0,001)$

Fonte: SUVIS Capela do Socorro.
Tabela 3. Surtos de varicela por mês de ocorrência, Capela do Socorro, 2005

\begin{tabular}{lcc}
\hline Mês & Surtos & Número de casos \\
\hline Janeiro & 01 & 37 \\
Fevereiro & 02 & 24 \\
Março & 01 & 07 \\
Abril & 01 & 34 \\
Maio & 05 & 101 \\
Junho & 10 & 149 \\
Julho & 06 & 108 \\
Agosto & 06 & 129 \\
Setembro & 02 & 17 \\
Outubro & 01 & 06 \\
Novembro & 0 & 0 \\
Dezembro & 01 & 05 \\
\hline Total & 36 & 617 \\
\hline Fonte: SUVIS Capela do Socorro.
\end{tabular}


Carvalho JZM et al. Surtos de varicela em creches na Capela do Socorro em 2005.

Tabela 4. Surtos de varicela por UBS mais próxima da creche, número de surtos e taxa de ataque, Capela do Socorro, 2005

\begin{tabular}{lcccc}
\hline UBS mais próxima & $\begin{array}{c}\text { Número de surtos } \\
\text { das creches }\end{array}$ & Total de alunos & $\begin{array}{c}\text { Total de casos } \\
\text { de varicela }\end{array}$ & Taxa de ataque \\
\hline Veleiros & 07 & 1078 & 108 & $10,01 \%$ \\
Jardim República & 06 & 1991 & 150 & $7,53 \%$ \\
Jardim Mirna & 05 & 934 & 60 & $21,41 \%$ \\
Ambulatório de Especialidades & & 306 & 28 & $9,15 \%$ \\
Dr. Milton Aldred & 03 & 777 & 29 & $3,73 \%$ \\
Ambulatório de Especialidades & 03 & 474 & 46 & $9,70 \%$ \\
$\quad$ Jardim Cliper & 03 & 574 & 91 & $15,85 \%$ \\
Jardim Castro Alves & 03 & 229 & 65 & $28,38 \%$ \\
Parque Residencial Cocaia & 02 & 137 & 16 & $10,94 \%$ \\
Jardim Eliane & 02 & 245 & 13 & $5,30 \%$ \\
Varginha & 01 & 100 & 11 & $0,11 \%$ \\
Jardim Icaraí Quintana & 01 & 6845 & 617 & $9,01 \%$ \\
Jordanópolis & 36 & & \\
\hline Total & & & \\
\hline Fon & & & & \\
\hline
\end{tabular}

Fonte: SUVIS Capela do Socorro.

Tabela 4a. Surtos de varicela em creches da UBS Veleiros, Capela do Socorro, 2005

\begin{tabular}{llccc}
\hline UBS-Veleiros & Casos & Não casos & Total de alunos & Taxa de ataque \\
\hline Escola Piemonte & 23 & 44 & 67 & $34,33 \%$ \\
Creche Guarapiranga & 8 & 115 & 123 & $6,50 \%$ \\
EMEI Clara Nunes & 34 & 322 & 356 & $9,55 \%$ \\
Creche Grão da vida & 4 & 158 & 162 & $2,47 \%$ \\
CEI Veleiros & 3 & 142 & 145 & $2,07 \%$ \\
CEI Nossa Senhora de Fátima & 28 & 74 & 102 & $27,45 \%$ \\
Creche Propame Assistencial & 8 & 115 & 123 & $6,50 \%$ \\
\hline Total & 108 & 970 & 1078 & $10,02 \%$ \\
\hline
\end{tabular}

$\mathrm{X}^{2}$ calc $=102.17 \quad(\mathrm{p}<0,0001)$

Fonte: SUVIS Capela do Socorro.

Tabela 4b. Surtos de varicela em creches da UBS Jd. República, Capela do Socorro, 2005

\begin{tabular}{llccc}
\hline Jd. República & Casos & Não casos & Total de alunos & Taxa de ataque \\
\hline CEI Cel. Geraldo de Arruda Penteado & 7 & 109 & 116 & $6,03 \%$ \\
Sociedade Beneficente Equilíbrio de Interlagos & 17 & 454 & 471 & $3,61 \%$ \\
CEI José Molina Jr. & 50 & 80 & 130 & $38,46 \%$ \\
Centro Social Comunitário Jd. Primavera & 41 & 183 & 224 & $18,30 \%$ \\
EMEI Sérgio Cardoso & 30 & 495 & 525 & $5,71 \%$ \\
EMEl Ângelo Kretã & 5 & 520 & 525 & $0,95 \%$ \\
\hline Total & 149 & 1842 & 1991 & $7,48 \%$ \\
\hline
\end{tabular}

$X^{2}$ calc $=261.72 \quad(p<0,0001)$

Fonte: SUVIS Capela do Socorro.

Tabela 4c. Surtos de varicela em creches da UBS Jd. Mirna, Capela do Socorro, 2005

\begin{tabular}{lcccc}
\hline Jd. Mirna & Casos & Não casos & Total de alunos & Taxa de ataque \\
\hline CEI Jd.Três corações & 3 & 166 & 169 & $1,78 \%$ \\
CEl Mitiko Matsushita Nevoeiro & 30 & 196 & 226 & $13,27 \%$ \\
Escola Recreativa Anjinho Feliz & 5 & 94 & 99 & $5,05 \%$ \\
Creche Caetano Etiene & 4 & 138 & 142 & $2,82 \%$ \\
CEU Três Lagos & 18 & 280 & 298 & $6,04 \%$ \\
\hline Total & 60 & 874 & 934 & $6,42 \%$ \\
\hline
\end{tabular}

$\mathrm{X}^{2}$ calc $=27.39 \quad(p<0,001)$

Fonte: SUVIS Capela do Socorro. 


\section{DISCUSSÃO}

A varicela não é doença notificável no Brasil, tornando difícil a estimativa de sua prevalência em nosso meio. No entanto, os surtos em creches, préescolas e comunidade em geral devem ser notificados ao Sistema Nacional de Agravos de Notificação (SINAN).

No presente estudo foram analisadas 36 instituições, cada uma com 1 surto de varicela, observando-se 617 casos em um total de 6845 alunos, com uma Taxa de Ataque de 9,01\%, como pode ser observado na Tabela 1. Comparando-se com os dados obtidos pelo Centro de Vigilância Epidemiológica "Professor Alexandre Vranjac" em São Jose do Rio Preto (SJRP), foram constatados 118 casos de varicela em um total de 3829 alunos, com uma Taxa de Ataque de 3,08\%.

Na região da Capela do Socorro, em número absoluto, percebe-se que a CEI José Molina Junior apresentou o maior número de casos entre todas as instituições. Considerando-se apenas os centros de educação infantil (CEI), de administração direta da prefeitura, observa-se que esta mesma CEI foi a que apresentou maior taxa de ataque $(38,46 \%)$, tendo sido acometidos 50 alunos em um total de 130, número bastante representativo. A CEI Núcleo III apresentou a segunda maior taxa (35,92\%).

Quando se comparam as escolas municipais de educação infantil, também de administração direta, nota-se uma taxa de ataque de 9,55\% na EMEI Clara Nunes, com 34 dos seus 356 alunos acometidos pela varicela. Já em relação às instituições conveniadas, preocupa muito a situação da Escola Piemonte, com taxa da ataque de $34,32 \%$ (23 alunos com varicela entre os 67 alunos), seguida pela creche AMA I Núcleo II com 22,22\%.

De acordo com o estudo feito em SJRP, encontrou-se 122 alunos com antecedentes de varicela, 57 com vacinação prévia e nenhum óbito. Já em nosso trabalho (Tabela 2), não ocorreu nenhum óbito, contabilizou-se 2383 casos que já haviam apresentado varicela anteriormente, e apenas 252 alunos com histórico de vacinação anterior contra esta doença. Tal fato deve ser considerado relevante, visto que a vacina contra varicela, apesar de bastante segura e eficaz, não está disponível na rede pública de saúde, sendo de difícil acesso às pessoas residentes na região da Capela do Socorro devido ao seu alto custo nas clínicas particulares de vacinação. Vale ressaltar que, em situações de surto, esta vacina é liberada para os suscetíveis de 1 a 4 anos pelo Centro de Referencia para Imunobiológicos Especiais e deve ser aplicada o mais rápido possível, na tentativa de bloqueio do mesmo.
A análise dos custos e benefícios da vacinação contra a varicela, de situações conseqüentes à moléstia, como tratamento médico e perda de dias de aula por parte dos alunos e de trabalho por parte dos responsáveis, e também da incidência cumulativa da doença em longo prazo nas faixas etárias de maior risco, considerando-se a vacinação em idades precoces, mostrou-se custo efetiva, resultando em benefícios a sua inclusão em programas de vacinação rotineira

Antes da ampla utilização da vacina, a varicela acometia aproximadamente quatro milhões de pessoas por ano nos Estados Unidos. Dados de vigilância de áreas sentinelas mostram que, desde 1995, ocorre uma dramática redução de incidência da doença (em torno de $90 \%$ ) e da mortalidade (cerca de 66\%). A maior queda ocorreu no grupo alvo primário da vacinação, crianças de 1 a 4 anos de idade.

$\mathrm{Na}$ Tabela 2a, pode ser observado que a faixa etária mais atingida entre os acometidos foi a de 1 a 4 anos, sendo esta também a mais populosa nos estabelecimentos avaliados, compreendendo 4073 crianças, das quais 524 tiveram varicela, com uma Taxa de Ataque de 12,87\%, significantemente maior em relação às demais $\left(x^{2}=195,50\right)$. Nesta faixa etária, cento e quarenta e sete crianças haviam recebido a vacina anteriormente, e 1302 já tinham apresentado a doença, o que não foi suficiente para abortar o surto de varicela porque 2100 crianças ainda estavam suscetíveis à infecção. Ressalta-se que a menor Taxa de Ataque $(0,97 \%)$ ocorreu na faixa etária de 6 a 9 anos, apresentando somente 11 casos. Já em SJRP a média de idade encontrada para o maior numero de casos foi de 2,85 anos.

Em relação ao mês de início dos casos, constata-se predomínio nos meses de inverno (junho, julho e agosto) com 22 surtos dos 36 , representando $61,11 \%$, como pode ser observado na Tabela 3 , sendo 149 , o maior número absoluto de casos relatados, no mês de junho. Nota-se que no mês de novembro não ocorreu nenhum surto. Tais dados diferem dos encontrados na literatura que refere predomínio dos casos de agosto a novembro.

Com relação às UBS (Tabela 4) houve maior número de surtos nas instituições próximas à UBS Veleiros (07), seguida da UBS Jardim República (06) e Jardim Mirna (05), tornando necessária uma análise mais aprofundada para verificar a maior concentração de casos nestas regiões, razão pela qual esta tabela foi desmembrada.

A UBS Veleiros está localizada no distrito do Socorro, região de maior poder aquisitivo em comparação aos demais distritos da região (Cidade Dutra e Grajaú). Nos estabelecimentos da UBS Veleiros (Tabela 4a), houve uma diferença estatisticamente significante entre Escola Piemonte e CEI Nossa Senhora 
de Fátima em relação às demais $\left(X^{2}=102,17\right)$. Considerando-se as instituições da área de abrangência da UBS Jd República (Tabela 4b), que se localiza no distrito da Cidade Dutra, nota-se que a CEI José Molina Junior apresentou Taxa de Ataque consideravelmente maior que a das outras instituições $(38,46 \%)$, sendo a EMEl Ângelo Kretã a que obteve a menor $(0,95 \%)$, dados estatisticamente significantes $\left(X^{2}=261,72\right)$.

Em relação à UBS Jd. Mirna (Tabela 4c), a CEI Mitiko Matsushita Nevoeiro, destacou-se com uma Taxa de Ataque de 13,27\%, também estatisticamente significante $\left(X^{2}=27,17\right)$.

\section{CONCLUSÃO}

Em virtude da grande transmissibilidade da doença, a mesma deveria constar como doença de notificação imediata, principalmente em creches, visando a diminuição de sua morbidade. Como decorrência desta notificação, teríamos maior êxito no controle do surto através da vacinação dos suscetíveis, minimizando assim a disseminação da doença. Concluímos também que a vacina contra esta moléstia deveria ser disponibilizada para toda a população, fazendo parte do esquema básico de vacinação e não só ser liberada apenas em situações de surto.

A varicela apresenta um custo econômico e social, em se manter afastada a criança de sua escola ou creche. Devido ao alto grau de transmissibilidade da varicela e da extrema rapidez com que a mesma pode se disseminar em ambientes confinados como creches, é que se fez necessário um estudo referente a surtos em creches como, por exemplo, as da Capela do Socorro.

Carvalho JZM, Rodrigues TR, Azzi TT, Burihan PCPR. Varicella outbreak in the Capela do Socorro, 2005. Rev Med (São Paulo). 2007 jul.-set.;86(3):148-54.

\begin{abstract}
The varicella disease is caused by a varicella-zoster virus. The varicella is extremely transmissible, being transmitted from person to person, its greater risk is on closed ambient as foundling-hospitals, class rooms and sick-rooms. The vaccine is insure and immune to children, healthy adults and in free compromising patients it shows good tolerability with few collateral reactions having an efficacy of $80 \%$. The goal of this work has being to analyze the varicella outbreak occurred in 2005 at Capela do Socorro region. The study done was transversal, through outbreak data ocurred in foundlings during the year of 2005 in the region Capela do Socorro Health Vigillancy Supervising (SUVIS). In the present study, 36 establishments have been analysed, having at least one varicella outbreak and showing a total of 617 cases out of a group of 6845 members, with an attack tax of $9,01 \%$. The data obtained has shown predominance on an age of $1-4$ years $\left(x^{2}=195.50\right)$ in the winter months $(61,11 \%$ of the outbreak) and a major outbreak numbers on near institutions to Veleiros, Republica and Mirna, respectively. The CEI José Molina has been the one that presented major attack tax $(38,46 \%)$. Due to the great transmissibility of varicella and to the extreme rapidity that it can be disseminated in confined ambients as foundlings, is the reason of the importance of a study related to the foundlings as those of the Capela do Socorro.
\end{abstract}

KEY WORDS: Disease outbreaks. Chickenpox/transmission. Child day care centers. Cross-sectional studies.

\section{REFERÊNCIAS}

1. Castiñeiras TM. Centro de Informação em Saúde para Viajantes [Citado 28 maio 2006]. Disponível em: http:// www.cives.ufrj.br.

2. McAdam JA, Sharp HA. Doenças infecciosas. In: Robbins \& Cotran. Patologia. 7a ed. São Paulo: Elsevier; 2005. p.383-4.

3. Nelson WE, McKay RJ, Vaughan VC. Infecções viróticas e infecções de origem presumidamente virótica: varicela. In: Vaughan III VC, McKay RJ, Nelsonm WE. Pediatria de Nelson. 10a ed. Rio de Janeiro: Interamericana; 1977. v.1, p.386-8: Varicela e Lupus-Zoster.

4. PRONAP. Infecções pelo vírus varicela-zoster: considera- ções diagnósticas e terapêuticas. São Paulo: SBP; 2001. extra1, p.64-5.

5. São Paulo (Estado). Secretaria de Estado da Saúde. Caxumba e varicela: orientação para surtos e epidemias. São Paulo: CVE; 2001. p.14-23.

6. São Paulo (Estado). Secretaria de Estado da Saúde. Surto de varicela em creches e escolas da Direção Regional de Saúde XXII, junho 2005. São Paulo. Rev Saude Publica. 2005;39:687-90.

7. Siegel S, Castellan NJ Jr. Nonparametrics, statistics. 2nd ed. New York: McGraw-Hill; 1988. 